Received Date : 20-Apr-2015

Revised Date : 30-May-2016

Accepted Date : 02-Jul-2016

Article type : Original Article - Kidney
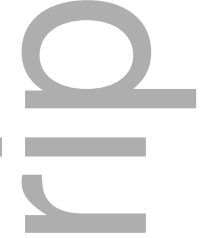

Renal cellular hypoxia in adenine-induced chronic kidney disease

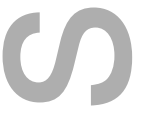

Debra Fong ${ }^{1}$, Md Mahbub Ullah ${ }^{1}$, Jaswini G. Lal ${ }^{1}$, Amany Abdelkader ${ }^{1}$, Connie P.C. Ow ${ }^{1}$, Lucinda M. Hilliard ${ }^{1}$, Sharon D. Ricardo ${ }^{2}$, Darren J. Kelly ${ }^{3}$

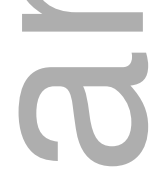

\& Roger G. Evans ${ }^{1}$

Biomedicine Discovery Institute and Departments of ${ }^{1}$ Physiology and ${ }^{2}$ Anatomy and Developmental Biology, Monash University, Clayton, Melbourne, Australia.

${ }^{3}$ Department of Medicine, University of Melbourne, St Vincent's Hospital, Fitzroy,

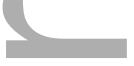
Melbourne, Australia.

Running title: Hypoxia in adenine-induced CKD

\title{
Conflict of Interest
}

None

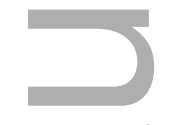

This is the author manuscript accepted for publication and has undergone full peer review but has not been through the copyediting, typesetting, pagination and proofreading process, which may lead to differences between this version and the Version of Record. Please cite this article as doi: 10.1111/1440-1681.12621

This article is protected by copyright. All rights reserved 


\section{Author for correspondence:}

Dr Roger Evans

Department of Physiology

PO Box 13F, Monash University, Victoria 3800, Australia

Tel: 61399051466

Fax: 61399052566

Email: Roger.Evans@monash.edu

\section{Abstract}

We determined whether adenine-induced chronic kidney disease (CKD) in rats is associated with renal tissue hypoxia. Adenine $(100 \mathrm{mg})$ or its vehicle was administered to male SpragueDawley rats, daily by oral gavage, over a 15 day period. Renal function was assessed before and 7 and 14 days after adenine treatment commenced, by collection of a $24 \mathrm{~h}$ urine sample and a blood sample from the tail vein. On day 15, arterial pressure was measured in conscious rats via the tail artery. Renal tissue hypoxia was then assessed by pimonidazole adduct immunohistochemistry and fibrosis was assessed by staining tissue with picrosirius red and Masson's trichrome. CKD was evident within 7 days of commencing adenine treatment, as demonstrated by increased urinary albumin to creatinine ratio (30 \pm 12 -fold). By day 14 of adenine treatment plasma creatinine concentration was more than 7-fold greater, and plasma urea more than 5-fold greater, than their baseline levels. On day 15, adenine-treated rats had slightly elevated mean arterial pressure $(8 \mathrm{mmHg})$, anaemia and renomegaly. Kidneys of adenine-treated rats were characterised by the presence of tubular casts, dilated tubules, expansion of the interstitial space, accumulation of collagen, and tubulointerstitial hypoxia. Pimonidazole staining (hypoxia) co-localised with fibrosis and was present in both patent and occluded tubules. We conclude that renal tissue hypoxia develops rapidly in adenine-induced CKD. This model, therefore, should prove useful for examination of the temporal and spatial relationships between tubulointerstitial hypoxia and the development of $\mathrm{CKD}$, and thus the testing of the 'chronic hypoxia hypothesis'.

\section{Abstract word count $=249$}


Keywords: fibrosis, pimonidazole, hypoxia

\section{Introduction}

Renal tissue hypoxia has been demonstrated in multiple forms of chronic kidney disease (CKD), including polycystic kidney disease, ${ }^{1-3}$ diabetic nephropathy, ${ }^{4}$ the remnant kidney model, ${ }^{5}$ and CKD following ischaemia-reperfusion injury. ${ }^{6,7}$ This has led to the proposition that hypoxia represents a final common pathway for the development of multiple forms of $\mathrm{CKD} ;{ }^{8}$ the so-called "chronic hypoxia hypothesis'. ${ }^{9}$ More recent observations have even provided evidence that renal hypoxia per se might be sufficient to initiate the development of CKD. ${ }^{10}$

The relatively slow development of injury and/or diminished glomerular filtration rate (GFR) in most rodent models of CKD has made it difficult to tease out the causal relationships between tubulointerstitial hypoxia and the pathogenesis of nephropathy. For example, after recovery from acute ischaemia-reperfusion injury, there is little evidence of renal functional abnormalities or abnormal tubular morphology 8 weeks after the initial insult and only mild proteinuria is observed after 16 weeks. ${ }^{11}$ In most rodent models of polycystic kidney disease, there is some evidence of abnormalities in renal tissue structure at birth, but then many weeks or months are required for the condition to progress to frank CKD. ${ }^{12,13}$ Furthermore, in type 1 diabetes, induced by streptozotocin in rats, only relatively mild tissue damage is observed in the first month after the induction of diabetes. ${ }^{14}$ Even in the remnant kidney model, which has been extensively used in studies of the pathogenesis of $\mathrm{CKD}$, nephropathy develops over weeks rather than days. ${ }^{5}$

In order for us to test the chronic hypoxia hypothesis in a definitive manner, it is vital that we are able to precisely define the temporal and spatial relationships between hypoxia and the progression of disease. ${ }^{15}$ That is, we must determine whether hypoxia occurs before, and in the same place as, tissue damage. Consequently, we require experimental models of CKD in which pathological changes in renal tissue develop rapidly in response to a defined stimulus. Administration of adenine, either in food ${ }^{16-25}$ or by oral gavage, ${ }^{26}$ has been shown to result in reproducible renal dysfunction. Adenine is metabolised to 2,8-dihydroxy-adenine which forms crystalline casts within the renal tubules. The formation of these casts is thought to initiate renal dysfunction. ${ }^{21} \mathrm{CKD}$ in adenine-treated rats is associated with hypertension and vascular calcification, renal interstitial fibrosis, proteinuria, increased water intake and urine flow, uraemia, and hyperkalaemia. ${ }^{25}$ Only modest increases in serum creatinine are observed after 2 weeks of adenine treatment, while more prolonged treatment results in marked renal This article is protected by copyright. All rights reserved 
pathology and markedly increased serum creatinine. ${ }^{17,18,20-22}$ In the current study, we tested the hypothesis that the early phase of adenine-induced CKD (i.e. after 2-weeks of treatment) is accompanied by renal tissue hypoxia, in order to evaluate the potential for this model to be used for testing the chronic hypoxia hypothesis.

\section{Results}

\section{Progression of chronic kidney disease}

Across the two weeks during which rats were treated with the vehicle for adenine, body weight increased $27 \pm 11 \%$, while food intake and faecal output slightly decreased (Table 1). Food intake and faecal output were consistently less in adenine-treated rats than vehicletreated rats. There were tendencies for adenine-treated rats to gain less weight than the vehicle-treated rats, although this apparent effect was not statistically significant (time*treatment interaction; $P=0.14$ ). In vehicle-treated rats, water intake and urine output gradually increased across the 14 days of the study, to be $28 \pm 18 \%$ and $116 \pm 57 \%$ greater than their baseline level at day 14, respectively. In contrast to vehicle-treated rats, much greater increases in water intake and urine output were observed in adenine-treated rats. Water intake had increased by $154 \pm 38 \%$ at day 7 and by $150 \pm 46 \%$ at day 14 . Urine output had increased by $346 \pm 83 \%$ at day 7 and by $372 \pm 96 \%$ at day 14 .

The rates of exeretion of sodium, chloride and osmolytes were indistinguishable in adeninetreated compared with vehicle-treated rats (Table 1). However, potassium excretion tended to increase in rats treated with adenine. This apparent effect was particularly evident on day 7 , when potassium excretion was $67 \%$ greater in adenine-treated rats than vehicle-treated rats. By day 7 of adenine-treatment urine osmolality was reduced by approximately $69 \pm 5 \%$ of its pre-treatment level. Proteinuria was also present at day 7 of adenine treatment. Urinary protein excretion had increased by $287 \pm 58 \%$ from its pre-treatment level and remained similarly increased at day 14 of adenine treatment. In contrast, the vehicle treated rats excreted little protein throughout the two week treatment period. In adenine-treated rats, urinary albumin excretion had increased $29 \pm 9$-fold and the urinary albumin to creatinine ratio had increased $30 \pm 12$ fold by day 7 of adenine treatment. Plasma creatinine, measured in three rats on day 0 , was below the level of detection (i.es $0.20 \mathrm{mg} / \mathrm{dL}$ ). By day 14 of adenine treatment it was more than 7 -fold greater $(1.50 \pm 0.13 \mathrm{mg} / \mathrm{dL}, \mathrm{n}=4)$. Plasma urea averaged $4.8 \pm 0.5 \mathrm{mM}(\mathrm{n}=3)$ on day 0 and $25.5 \pm 0.9 \mathrm{mM}(\mathrm{n}=3)$ on day 14 of adenine treatment. 
On the $15^{\text {th }}$ day of treatment, the body weight of adenine-treated rats was $16 \%$ less than that of vehicle treated rats (Table 2). Mean arterial pressure of adenine-treated rats averaged 8 $\mathrm{mmHg}$ higher than that of vehicle treated rats but heart rate did not differ significantly between the two groups. The kidneys of adenine-treated rats were $86 \%$ heavier than those of vehicle-treated rats. Haematocrit was less in adenine-treated rats $(31.9 \pm 3.2 \%)$ than in vehicle-treated rats $(38.2 \pm 1.4 \%)$.

\section{Histology and immunohistochemistry}

To visualise the tubular and vascular elements of the rat kidney, haematoxylin and eosin staining was performed. Kidneys of rats treated with vehicle (Figure 1a) were considerably smaller than the kidneys of adenine-treated rats (Figure 1e). Tissue architecture of the cortex and medulla in the kidneys of the rats treated with vehicle appeared normal (Figure 1a-d). The tubules were closely spaced and the interstitial space and blood vessels were not prominent. The glomeruli appeared regular with normal thickness of the basement membrane (Figure 1b). In contrast, the tissue architecture of the kidneys of rats treated with adenine was very different. There was considerable expansion of the interstitial space in the cortex and medulla (Figure 1e-h). The tubules appeared abnormal with irregular lumen space. The glomeruli were intact in their basement membrane but dilation of Bowman's space was seen (Figure 1f). The blood vessels appeared unaffected and similar to those of vehicle-treated rats. Characteristic tubular casts, presumably containing 2, 8-dihydroxyadenine, were also observed (Figure 2).

Tissues were stained with picrosirius red (Figure 3) and Masson's trichrome (Figure 4) to examine fibrosis. Masson's trichrome stains type I collagen as well as variety of matrix elements. ${ }^{27}$ In contrast, picro-sirius red is highly specific for type I and type III collagen. ${ }^{28}$ Sections of kidneys of vehicle-treated rats stained with picrosirius red appeared yellow and lacked red collagen positive stain (Figure 3a). The only staining observed was in the perivascular adventitia and around the glomeruli (Figure 3b). In contrast, there was extensive picrosirius red staining in the kidney of the adenine-treated rats (Figure 3e). Considerable staining, denoting collagen deposition, was observed in the expanded interstitial space (Figure 3f-h). Regarding Masson's trichrome staining, there was little blue staining (reflecting fibrosis) in sections from vehicle-treated rats (Figure $4 \mathrm{~b}$ and Figure 4c), except for the expected perivascular staining around intrarenal arteries. In contrast, there was considerable positive staining in the cortex, outer medulla and inner medulla of adeninetreated rats (Figure 4f-h). Quantification of Masson's trichrome staining revealed more than This article is protected by copyright. All rights reserved 
3-fold greater staining in the cortex and medulla of adenine-treated rats compared to vehicletreated rats (Figure 5).

Pimonidazole immunohistochemistry was performed to detect the presence of hypoxia. In the kidneys of all vehicle-treated rats, positive staining, denoting hypoxia, was mainly seen in the thick ascending limb and collecting ducts in the outer and inner medulla respectively (Figure 6a-d). Very little apparent staining was seen in the cortex of all of the vehicle-treated rats (Figure 6b). In contrast, considerable staining was observed throughout the entire kidney of all rats that were treated with adenine (Figure 6e). The degree of pimonidazole staining was markedly greater in the cortex of adenine-treated rats, particularly in the epithelial lining of tubules, when compared to vehicle-treated rats. There was also considerable staining seen in the outer and inner medulla (Figure 6g-h).

It was noted that some tubules in the cortex of adenine-treated rats were patent or even dilated, while others had a closed lumen. This phenomenon likely arises from the formation of tubular casts (Figure 2). We reasoned that tubules with an open lumen may consume more oxygen, for reabsorption of sodium, than tubules with a closed lumen. We hypothesised that the resultant heterogeneity of tubular oxygen consumption might lead to heterogeneity in renal hypoxia. However, the proportion of open tubules that stained positive for pimonidazole adducts was similar to the proportion of positive stained closed tubules (Figure 7).

The co-localisation of hypoxia and fibrosis was examined in serial sections stained for pimonidazole adducts and with Masson's trichrome (Figure 8). Hypoxia and fibrosis were ubiquitously distributed in kidneys of adenine-treated rats. It was not possible to identify areas of the kidney that were hypoxic but not fibrotic, or fibrotic but not hypoxic.

\section{Discussion}

We found that 15 days of administration of adenine resulted in marked tubulointerstital hypoxia associated with fibrosis, along with profound renal dysfunction Thus, this experimental model may be useful for teasing out the temporal and spatial relationships between tubulointerstitial hypoxia, fibrogenesis, and the initiation and progression of CKD.

CKD is usually defined as a gradual loss of renal function, as quantified by measurement of GFR or plasma or serum concentrations of creatinine. According to this definition, the rats This article is protected by copyright. All rights reserved 
we studied had severe CKD by day 14 of adenine-treatment, as reflected by a more than 7fold increase in plasma creatinine and 5-fold increase in plasma urea. Our findings are consistent with those of previous studies in which adenine has been administered for longer periods (i.e. 4 or more weeks). ${ }^{17,18,20-22}$ It is well established that creatinine clearance overestimates glomerular filtration rate in rats, principally due to tubular secretion of creatinine. ${ }^{29}$ Nevertheless, between-subject and within-subject differences in plasma creatinine concentration usually reflect differences in GFR. ${ }^{3}$

Pathological changes were detectable at the tissue level after two-weeks of adenine treatment, particularly in the form of interstitial fibrosis and tubular casts. There were also marked increases in urinary albumin and total protein excretion. There was a marked increase in urine flow and reduced urinary osmolarity. Reduced urinary concentrating ability is a hallmark of CKD in humans ${ }^{30}$ and animal models (e.g. polycystic kidney disease ${ }^{2}$ ). However, we cannot discount the possibility that the effect of adenine-treatment on urinary osmolarity was caused by increased thirst as opposed to reduced urinary concentrating ability.

Multiple mechanisms likely contribute to the development of renal tissue hypoxia during adenine-treatment. Our current findings indicate development of renal fibrosis in this model that is co-localized with hypoxia. Indeed, in serial sections, we were unable to identify regions of the kidneys of adenine-treated rats that stained positive for pimonidazole but not the blue stain of Masson's trichrome, or vice versa. Tubulointerstitial fibrosis increases the barriers to diffusion of oxygen from the vascular to the tubulointerstitial compartment. ${ }^{9,}, 31$ Thus, fibrosis likely makes some direct contribution to the development of hypoxia in adenine-treated rats. There is also strong evidence that, in other models of CKD such as renovascular disease, fibrosis drives rarefaction of capillaries which in turn would be expected to reduce oxygen delivery to tissue. ${ }^{32}$ We are not aware of any published reports of renal vascular structure in adenine-induced CKD. However, Milman and colleagues recently provided evidence of blunted perfusion and renal vascular reactivity in anaesthetised mice with adenine-induced CKD, using a magnetic resonance imaging technique for assessing the renal vascular response to hyperoxia. ${ }^{24}$ Consistent with these observations, renal blood flow measured directly by transit-time ultrasound flowmetry ${ }^{33}$ or electromagnetic flowmetry ${ }^{34}$ under anaesthesia, and by the clearance of para-aminohippurate in the conscious state, ${ }^{35}$ has been observed to be less in rats with adenine-induced CKD compared to control animals. Thus, it is likely that abnormalities in both renal vascular structure and function contribute to the development of tissue hypoxia in adenine-treated rats. It is also likely that anaemia This article is protected by copyright. All rights reserved 
contributes to the development of renal hypoxia, since haematocrit was considerably less after 15 days of adenine-treatment than after vehicle treatment. This observation is also consistent with those arising from previous studies using this model. ${ }^{33}$

According to the chronic hypoxia hypothesis developed by Fine and colleagues, one of the major drivers of tubulointerstitial hypoxia in CKD is hyperfiltration in remnant functional nephrons. ${ }^{36}$ This is thought to be a consequence of increased tubular sodium reabsorption, the major source of oxygen consumption in the kidney. ${ }^{37}$ We reasoned that this might lead to localisation of hypoxia selectively within patent tubules in the kidneys of adenine-treated rats and the presence of less severe hypoxia in those tubules that are occluded due to the presence of upstream tubular casts. Our use of perfusion fixation in the current study allowed us to examine this proposition, since we could clearly distinguish between patent and occluded tubules. We could not detect differences in the proportion of tubular profiles that stained positive for pimonidazole, according to whether the tubules were occluded or patent. Thus, our current findings do not provide support this aspect of the chronic hypoxia hypothesis. However, we must acknowledge an important limitation of pimonidazole adduct immunohistochemistry using 3, 3'-diaminobenzidine (DAB). DAB cannot be quantified because it does not obey the Beer-Lambert law. ${ }^{38}$ Thus, future studies should examine the impact of tubular patency on renal tissue oxygenation in greater detail through use of quantitative methods for analysis of hypoxia at the cellular level.

Renal fibrosis in adenine-treated rats is also likely to be driven by multiple factors. The role of epithelial-to-mesenchymal transition in the development of fibrosis in CKD remains controversial. ${ }^{39}$ Nevertheless, there are a number of pieces of evidence that support a role for this mechanism in adenine-treated rodents. For example, Wang et al demonstrated upregulation of the pro-fibrogenic factors transforming growth factor (TGF)- $\beta 1$ and TGF- $\beta 2$, together with down-regulation of the (anti-fibrotic) translational repressors of TGF- $\beta 2$, micro-RNA (miR) miR200a and miR-141, in mice with adenine-induced CKD. ${ }^{26}$ The authors provided evidence from cell-culture studies that TGF- $\beta 1$ and TGF- $\beta 2$ can drive epithelial-tomesenchymal transition and so fibrogenesis. On the other hand, Wu et al recently provided evidence for a role of endothelial-to-mesenchymal transition, driven by elevated circulating levels of parathyroid hormone, in fibrogenesis in adenine-induced CKD. ${ }^{23}$ Interestingly, they were able to inhibit both endothelial-to-mesenchymal transition and the development of fibriosis with the calcimetic cinacalcet. 
Hypoxia, through increased signalling via hypoxia-inducible factors (HIFs), may also play an important role in the development of fibrosis in $\mathrm{CKD} .{ }^{40}$ For example, renal fibrosis in rats with two-kidney-one-clip hypertension can be blunted by silencing HIF- $1 \alpha^{41}$ Indeed, HIF activation and TGF $\beta$ signalling may be able to induce fibrosis even in the absence of hypoxia. ${ }^{42}$ The role of hypoxia and HIFs in driving fibrosis in adenine-induced CKD has not, to our knowledge, been directly assessed. Nevertheless, it remains possible that a vicious cycle may develop whereby hypoxia drives fibrosis and fibrosis drives hypoxia, as has been postulated to occur in other forms of CKD. ${ }^{15,36}$

Our current finding of marked proteinuria in adenine-treated rats, as early as one week after commencing treatment, together with the documentation of both fibrosis and cellular hypoxia after 2 weeks, provides the basis for examination of the role of hypoxia in the development of nephropathy in this model. In particular, it should be possible to use radiotelemetry, in conscious rats, to precisely define the time-course of the development of renal tissue hypoxia. ${ }^{43}$ It should also be possible to define the relative contributions of anaemia, renal ischemia, and altered renal oxygen consumption in the development of this hypoxia. ${ }^{3,7} \mathrm{~A}$ major challenge will be to find ways to ameliorate hypoxia in adenine-induced CKD to determine whether such treatments can blunt the development of renal dysfunction. Exposure of adenine-treated rats to hyperoxia is one possibility. However, the efficacy of such treatments will be limited both by the potential for oxygen toxicity ${ }^{44}$ and by loss of oxygen in the pre-glomerular circulation. ${ }^{45,46}$ Anti-fibrotic therapy might also be a useful approach to break the putative vicious cycle of hypoxia and fibrosis. ${ }^{47}$

In conclusion, our current findings indicate that severe renal dysfunction, accompanied by hypoxia and fibrosis, develops within two-weeks of adenine-treatment. Therefore, in the future, this model should prove useful for testing the chronic hypoxia hypothesis for the development of CKD.

\section{Methods}

\section{Animals}

Eighteen male Sprague-Dawley rats (Monash Animal Research Platform, Monash University, Melbourne, VIC, Australia), weighing on average $214 \pm 5 \mathrm{~g}$, were used in this study. At the commencement of treatment, the rats were 7 weeks of age. The rats were housed under This article is protected by copyright. All rights reserved 
standard laboratory conditions; $22 \pm 1^{\circ} \mathrm{C}, 40 \pm 1 \%$ humidity and with a $12 \mathrm{~h}$ light/dark cycle $(6 \mathrm{am}-6 \mathrm{pm})$. Tap water and standard rat chow were available ad libitum. All experimental procedures were approved by the Animal Ethics Committee of the School of Biomedical Sciences, Monash University, and were in accordance with the Australian Code of Practice for the Care and Use of Animals for Scientific Purposes. Rats were housed individually and allowed 1 week to acclimatise to these conditions before entering the study protocol.

\section{Experimental protocol}

Adenine-induced chronic kidney disease: All rats were treated daily with either adenine (100 mg; Sigma-Aldrich, Castle Hill, NSW, Australia) or its vehicle $(1 \mathrm{~mL}$ of $0.5 \% \mathrm{w} / \mathrm{v}$ methylcellulose; Sigma-Aldrich) by oral gavage for a period of 15 days. The experiments were performed in two series. In the first series, 6 adenine-treated rats and 6 vehicle-treated rats were studied as described below $(165-252 \mathrm{~g})$. For technical reasons, we were unable to obtain sufficient blood from most of these animals for measurement of plasma creatinine concentration. Therefore, an additional cohort of rats (192-212 g) were included in a supplementary study ( $n=3$ per group). The rats were treated identically to those in the first series, but the only analysis performed was measurement of plasma creatinine concentration.

Metabolic studies: Rats were housed individually in metabolic cages for 24 hours before, and 7 and 14 days after, commencement of the treatments (metabolic studies). Consumption of food and water and production of faeces and urine were measured over the $24 \mathrm{~h}$ period. A day prior to the first metabolic study, the rats underwent a 6-hour training period for acclimatisation. Urine samples were transferred into several $1 \mathrm{ml}$ aliquots and stored at $20^{\circ} \mathrm{C}$ for later analysis. At the completion of the $24 \mathrm{~h}$ period in the metabolic cage a $0.5-1 \mathrm{ml}$ blood sample was collected from the tail vein from the conscious rat. Blood was centrifuged at $3000 \mathrm{~g}$ at $4^{\circ} \mathrm{C}$ for 10 minutes and the plasma stored in $50 \mu \mathrm{L}$ aliquots at $-20^{\circ} \mathrm{C}$ for later analysis.

Measurement of arterial pressure and tissue processing: On day 15 of the experimental protocol, arterial pressure was measured via the tail artery. The tail artery was catheterised under anaesthesia with isoflurane (1-4\% v/v in $100 \% \mathrm{O}_{2}$, Isoflo, Baxter Healthcare, Old Toongabbie, NSW, Australia). Following a $60 \mathrm{~min}$ recovery period, with the rats fully conscious, the catheter was connected to a pressure transducer (Cobe, Arvada, CO, USA) and bridge amplifier (QA1, Scientific Concepts, Mount Waverley, VIC, Australia) interfaced with a computer running a LabView-based data acquisition program (Universal Acquisition, 
University of Auckland, New Zealand). After a 5-10 min equilibration period, mean arterial pressure and heart rate were recorded for $30 \mathrm{~min}$ in the conscious rat.

After measurement of arterial pressure, the hypoxic marker 2-pimonidazole $(60 \mathrm{mg} / \mathrm{kg}$ pimonidazole hydrochloride, Hypoxyprobe Inc, Burlington, MA, USA) was administered intraperitoneally. Sixty minutes later, each rat was anaesthetised with pentobarbitone sodium (60 mg/kg, i.p., Sigma-Aldrich). Once a surgical level of anaesthesia was reached, a midline incision was made to expose the abdominal aorta and inferior vena cava. The left kidney was then perfusion fixed with $50-100 \mathrm{ml} 4 \% \mathrm{w} / \mathrm{v}$ paraformaldehyde (PFA, Sigma-Aldrich), weighed and decapsulated, and post-fixed in a $4 \%$ PFA solution at $4{ }^{\circ} \mathrm{C}$ overnight.

\section{Analytical methods}

Analysis of urine and plasma samples: Urinary electrolyte ( $\mathrm{Na}+, \mathrm{K}+$ and $\mathrm{Cl}-)$ concentration was measured using an ion analyser (Rapidchem, Siemens Healthcare Diagnostics Pty Ltd, Bayswater, VIC, Australia). Urine osmolarity was measured by freezing point estimation (Model 2020, Advanced Instruments, Inc., John Morris Scientific Pty Ltd, Chatswood, NSW, Australia). Urinary albumin concentration was determined using an enzyme-linked immunosorbent assay (Rat Albumin ELISA Kit, Bethyl Laboratories Inc, Montgomery, TX, USA). Urinary creatinine concentration was measured using a creatinine assay kit (Cayman Chemical Company, Ann Arbor, MI, USA). Urinary protein concentration was measured by the Bradford method (Bio-Rad Laboratories, USA). ${ }^{48}$ Plasma creatinine and urea were measured using a point-of-care device (iSTAT, Chem8+ Cartridge, Abbot Laboratories, Abbott Park, IL, USA).

Histology and immunohistochemistry: Two sections from each kidney were stained with haematoxylin and eosin, ${ }^{7}$ Masson's trichrome, ${ }^{43}$ or picrosirius red, ${ }^{2}$ or processed for pimonidazole adduct immunohistochemistry ${ }^{7}$ as previously described. Masson's trichrome stains type I collagen as well as variety of matrix elements, ${ }^{27}$ whereas picrosirius red is highly specific for type I and type III collagen. ${ }^{28}$

Additionally, two sets of serial sections from each kidney were processed so that adjacent sections could be stained with Masson's trichrome ${ }^{43}$ and for pimonidazole adducts. ${ }^{7}$ All sections were scanned using Scan Scope (Aperio, Vista, CA). Quantification of fibrosis in sections processed for Masson's trichrome staining was performed using the Image Scope Positive Pixel Count Algorithm (Version 9, Aperio). An additional analysis was performed to 
determine whether hypoxia was associated with the state of patency of individual tubules, For this analysis, 20 open (patent) tubules and 20 closed (occluded) tubules were identified in the cortex of each kidney section stained with Masson's trichrome from six adenine-treated rats. These tubules were then identified in the adjacent sections stained for pimonidazole adducts and scored for either the presence or absence of pimonidazole staining.

\section{Statistical analysis}

All data are expressed as mean \pm sem. Hypothesis testing was performed using the software package SYSTAT (Version 13, Systat Software Inc, Chicago, IL, USA). Measurements obtained before and 7 and 14 days after commencing treatment with adenine or its vehicle were subjected to repeated measures analysis of variance. $P$ values derived from the withinsubjects factor 'time' were conservatively adjusted using the Greenhouse-Geisser correction. ${ }^{49}$ Measurements obtained on day 15 of treatment with adenine or its vehicle were subjected to Student's unpaired t-test. Two sided $P \leq 0.05$ was considered statistically

\section{Acknowledgements}

This work was supported by grants from the National Health and Medical Research Council of Australia (606601 \& 1024575).

\section{References}

1. Bernhardt WM, Wiesener MS, Weidemann A, et al. Involvement of hypoxiainducible transcription factors in polycystic kidney disease. Am J Pathol 2007; 170:830-42.

2. Ding A, Kalaignanasundaram P, Ricardo SD, et al. Chronic treatment with tempol does not significantly ameliorate renal tissue hypoxia or disease progression in a rodent model of polycystic kidney disease. Clin Exp Pharmacol Physiol 2012; 39:917-29.

3. Ow CP, Abdelkader A, Hilliard LM, Phillips JK, Evans RG. Determinants of renal tissue hypoxia in a rat model of polycystic kidney disease. Am J Physiol Regul Integr Comp Physiol 2014; 307:R1207-15.

4. Palm F, Cederberg J, Hansell P, Liss P, Carlsson PO. Reactive oxygen species cause diabetes-induced decrease in renal oxygen tension. Diabetologia 2003; 46:1153-60.

5. Manotham K, Tanaka T, Matsumoto M, et al. Evidence of tubular hypoxia in the early phase in the remnant kidney model. J Am Soc Nephrol 2004; 15:1277-88.

6. Basile DP, Donohoe DL, Roethe K, Mattson DL. Chronic renal hypoxia after acute ischemic injury: effects of L-arginine on hypoxia and secondary damage. Am J Physiol Renal Physiol 2003; 284:F338-48.

This article is protected by copyright. All rights reserved 
7. Abdelkader A, Ho J, Ow CP, et al. Renal oxygenation in acute renal ischemiareperfusion injury. Am J Physiol Renal Physiol 2014; 306:F1026-F38.

8. Mimura I, Nangaku M. The suffocating kidney: tubulointerstitial hypoxia in end-stage renal disease. Nat Rev Nephrol 2010; 6:667-78.

9. Fine LG, Norman JT. Chronic hypoxia as a mechanism of progression of chronic kidney diseases: from hypothesis to novel therapeutics. Kidney Int 2008; 74:867-72.

10. Friederich-Persson M, Thorn E, Hansell P, Nangaku M, Levin M, Palm F. Kidney hypoxia, attributable to increased oxygen consumption, induces nephropathy independently of hyperglycemia and oxidative stress. Hypertension 2013; 62:914-9.

11. Basile DP, Donohoe D, Roethe K, Osborn JL. Renal ischemic injury results in permanent damage to peritubular capillaries and influences long-term function. Am J Physiol Renal Physiol 2001; 281:F887-99.

12. Guay-Woodford LM. Murine models of polycystic kidney disease: molecular and therapeutic insights. Am J Physiol Renal Physiol 2003; 285:F1034-49.

13. Phillips JK, Hopwood D, Loxley RA, et al. Temporal relationship between renal cyst development, hypertension and cardiac hypertrophy in a new rat model of autosomal recessive polycystic kidney disease. Kidney Blood Press Res 2007; 30:129-44.

14. Kiran G, Nandini CD, Ramesh HP, Salimath PV. Progression of early phase diabetic nephropathy in streptozotocin-induced diabetic rats: evaluation of various kidney-related parameters. Indian journal of experimental biology 2012; 50:133-40.

15. Evans RG, O'Connor PM. Initiation and progression of chronic kidney disease: can we definitively test the chronic hypoxia hypothesis? Hypertension 2013; 62:827-8.

16. Katsumata K, Kusano K, Hirata M, et al. Sevelamer hydrochloride prevents ectopic calcification and renal osteodystrophy in chronic renal failure rats. Kidney Int 2003; 64:44150.

17. Nagano N, Miyata S, Abe M, et al. Effect of manipulating serum phosphorus with phosphate binder on circulating PTH and FGF23 in renal failure rats. Kidney Int 2006; 69:531-7.

18. Neven E, Dauwe S, De Broe ME, D'Haese PC, Persy V. Endochondral bone formation is involved in media calcification in rats and in men. Kidney Int 2007; 72:574-81. 19. Terai K, Mizukami K, Okada M. Comparison of chronic renal failure rats and modification of the preparation protocol as a hyperphosphataemia model. Nephrology (Carlton) 2008; 13:139-46. 
20. Matsui I, Hamano T, Mikami S, et al. Fully phosphorylated fetuin-A forms a mineral complex in the serum of rats with adenine-induced renal failure. Kidney Int 2009; 75:915-28. 21. Ikeda R, Imai Y, Maruyama W, Mizoguchi K. Systemic disorders of calcium dynamics in rats with adenine-induced renal failure: implication for chronic kidney diseaserelated complications. Nephrology (Carlton) 2010; 15:54-62.

22. Nguy L, Nilsson H, Lundgren $J$, et al. Vascular function in rats with adenine-induced chronic renal failure. Am J Physiol Regul Integr Comp Physiol 2012; 302:R1426-35.

23. Wu M, Tang RN, Liu H, Xu M, Pan MM, Liu BC. Cinacalcet attenuates the renal endothelial-to-mesenchymal transition in rats with adenine-induced renal failure. Am J Physiol Renal Physiol 2014; 306:F138-46.

24. Milman Z, Axelrod JH, Heyman SN, Nachmansson N, Abramovitch R. Assessment with Unenhanced MRI Techniques of Renal Morphology and Hemodynamic Changes during Acute Kidney Injury and Chronic Kidney Disease in Mice. Am J Nephrol 2014; 39:268-78. 25. Diwan V, Small D, Kauter K, Gobe GC, Brown L. Gender differences in adenineinduced chronic kidney disease and cardiovascular complications in rats. Am J Physiol Renal Physiol 2014; 307:F1169-78.

26. Wang B, Koh P, Winbanks C, et al. miR-200a Prevents renal fibrogenesis through repression of TGF-beta2 expression. Diabetes 2011; 60:280-7.

27. Lillie RD. Further experiments with the Masson trichrome modification of Mallory's connective tissue stain Stain Technology 1940; 15:17-22.

28. Junqueira LC, Cossermelli W, Brentani R. Differential staining of collagens type I, II and III by Sirius Red and polarization microscopy. Archivum histologicum Japonicum = Nihon soshikigaku kiroku 1978; 41:267-74.

29. Darling IM, Morris ME. Evaluation of "true" creatinine clearance in rats reveals extensive renal secretion. Pharmaceutical research 1991; 8:1318-22.

30. Perucca J, Bouby N, Valeix P, Bankir L. Sex difference in urine concentration across differing ages, sodium intake, and level of kidney disease. Am J Physiol Regul Integr Comp Physiol 2007; 292:R700-5.

31. Nangaku M. Chronic hypoxia and tubulointerstitial injury: a final common pathway to end-stage renal failure. J Am Soc Nephrol 2006; 17:17-25.

32. Chade AR. Renal vascular structure and rarefaction. Compr Physiol 2013; 3:817-31.

33. Saeed A, Dibona GF, Grimberg E, et al. High-NaCl diet impairs dynamic renal blood flow autoregulation in rats with adenine-induced chronic renal failure. Am J Physiol Regul Integr Comp Physiol 2014; 306:R411-9.

This article is protected by copyright. All rights reserved 
34. Johns EJ, Rutkowski B. The action of atriopeptin III on renal function in two models of chronic renal failure in the rat. Br J Pharmacol 1990; 99:317-22.

35. Ienaga K, Yokozawa T. Treatment with NZ-419 (5-Hydroxy-1-methylimidazoline2,4-dione), a novel intrinsic antioxidant, against the progression of chronic kidney disease at stages 3 and 4 in rats. Biol Pharm Bull 2010; 33:809-15.

36. Fine LG, Bandyopadhay D, Norman JT. Is there a common mechanism for the progression of different types of renal diseases other than proteinuria? Towards the unifying theme of chronic hypoxia. Kidney Int Suppl 2000; 75:S22-6.

37. Evans RG, Harrop GK, Ngo JP, Ow CP, O'Connor PM. Basal renal oxygen consumption and the efficiency of oxygen utilization for sodium reabsorption. Am J Physiol Renal Physiol 2014; 306:F551-F60.

38. van der Loos CM. Multiple immunoenzyme staining: methods and visualizations for the observation with spectral imaging. The journal of histochemistry and cytochemistry: official journal of the Histochemistry Society 2008; 56:313-28.

39. Grabias BM, Konstantopoulos K. The physical basis of renal fibrosis: effects of altered hydrodynamic forces on kidney homeostasis. Am J Physiol Renal Physiol 2014; 306:F473-85.

40. Higgins DF, Kimura K, Bernhardt WM, et al. Hypoxia promotes fibrogenesis in vivo via HIF-1 stimulation of epithelial-to-mesenchymal transition. J Clin Invest 2007; 117:381020.

41. Wang Z, Zhu Q, Li PL, et al. Silencing of hypoxia-inducible factor-1alpha gene attenuates chronic ischemic renal injury in two-kidney, one-clip rats. Am J Physiol Renal Physiol 2014; 306:F1236-42.

42. Hanna C, Hubchak SC, Liang X, et al. Hypoxia-inducible factor-2alpha and TGF-beta signaling interact to promote normoxic glomerular fibrogenesis. Am J Physiol Renal Physiol 2013; 305:F1323-31.

43. Koeners MP, Ow CP, Russell DM, et al. Telemetry-based oxygen sensor for continuous monitoring of kidney oxygenation in conscious rats. Am J Physiol Renal Physiol 2013; 304:F1471-80.

44. Freeman BA, Crapo JD. Hyperoxia increases oxygen radical production in rat lungs and lung mitochondria. J Biol Chem 1981; 256:10986-92.

45. Schurek HJ, Jost U, Baumgartl H, Bertram H, Heckmann U. Evidence for a preglomerular oxygen diffusion shunt in rat renal cortex. Am J Physiol 1990; 259:F910-5. 
46. Evans RG, Gardiner BS, Smith DW, O'Connor PM. Intrarenal oxygenation: unique challenges and the biophysical basis of homeostasis. Am J Physiol Renal Physiol 2008; 295:F1259-70.

47. Kelly DJ, Zhang Y, Gow R, Gilbert RE. Tranilast attenuates structural and functional aspects of renal injury in the remnant kidney model. J Am Soc Nephrol 2004; 15:2619-29. 48. Bradford MM. A rapid and sensitive method for the quantitation of microgram quantities of protein utilizing the principle of protein-dye binding. Analytical biochemistry 1976; 72:248-54.

49. Ludbrook J. Repeated measurements and multiple comparisons in cardiovascular research. Cardiovasc Res 1994; 28:303-11.

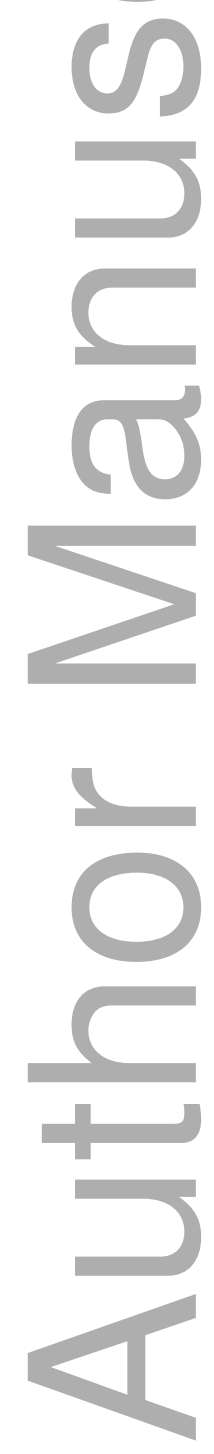


Table 1: Characteristics of adenine-treated and vehicle-treated rats across the course of the first 14 days of the experiment.

\begin{tabular}{|c|c|c|c|c|c|c|c|c|c|c|c|}
\hline \multirow{2}{*}{ Variable } & \multicolumn{4}{|c|}{ Vehicle-treated rats } & \multicolumn{4}{|c|}{ Adenine-treated rats } & \multicolumn{3}{|c|}{$\begin{array}{c}P \text { values from Repeated } \\
\text { Measures ANOVA }\end{array}$} \\
\hline & $\mathbf{n}$ & Day 0 & Day 7 & Day 14 & $\mathbf{n}$ & Day 0 & Day 7 & Day 14 & Treat & Time & Interaction \\
\hline \multicolumn{12}{|c|}{ Fluid and Energy Balance } \\
\hline Body Weight (g) & 6 & $215 \pm 13$ & $256 \pm 9$ & $269 \pm 13$ & 6 & $221 \pm 10$ & $248 \pm 15$ & $236 \pm 13$ & 0.43 & 0.003 & 0.14 \\
\hline Food Intake (g/day) & 6 & $20.1 \pm 0.6$ & $15.4 \pm 2.3$ & $18.4 \pm 1.3$ & 6 & $19.7 \pm 0.5$ & $12.8 \pm 2.0$ & $11.2 \pm 1.4$ & 0.04 & 0.003 & 0.08 \\
\hline Faeces Output (g/day) & 6 & $10.0 \pm 0.4$ & $6.9 \pm 1.3$ & $7.1 \pm 1.0$ & 6 & $8.2 \pm 0.5$ & $5.1 \pm 0.6$ & $5.3 \pm 1.1$ & $\mathbf{0 . 0 3}$ & 0.004 & 0.99 \\
\hline Water Intake (ml/day) & 6 & $23.3 \pm 2.1$ & $23.3 \pm 4.0$ & $29.2 \pm 3.7$ & 6 & $23.3 \pm 3.3$ & $54.2 \pm 5.2$ & $54.2 \pm 7.8$ & 0.001 & 0.002 & 0.009 \\
\hline Urine Output (ml/day) & 6 & $8.0 \pm 0.8$ & $11.3 \pm 1.9$ & $15.1 \pm 2.8$ & 6 & $11.1 \pm 2.7$ & $40.1 \pm 4.3$ & $43.0 \pm 6.0$ & $<0.001$ & $<0.001$ & 0.003 \\
\hline \multicolumn{12}{|l|}{ Electrolyte Excretion } \\
\hline Sodium $(\mu \mathrm{mol} / \mathrm{min})$ & 6 & $0.80 \pm 0.14$ & $0.75 \pm 0.10$ & $0.77 \pm 0.09$ & 6 & $0.89 \pm 0.07$ & $0.75 \pm 0.11$ & $0.71 \pm 0.12$ & 0.93 & 0.54 & 0.73 \\
\hline Chloride $(\mu \mathrm{mol} / \mathrm{min})$ & 6 & $1.38 \pm 0.21$ & $1.24 \pm 0.14$ & $1.57 \pm 0.10$ & 6 & $1.44 \pm 0.08$ & $1.60 \pm 0.13$ & $1.47 \pm 0.16$ & 0.38 & 0.64 & 0.30 \\
\hline Potassium $(\mu \mathrm{mol} / \mathrm{min})$ & 6 & $1.25 \pm 0.13$ & $1.07 \pm 0.13$ & $1.54 \pm 0.11$ & 6 & $1.29 \pm 0.13$ & $1.79 \pm 0.19$ & $1.78 \pm 0.26$ & $\mathbf{0 . 0 3}$ & 0.09 & 0.15 \\
\hline Osmolar ( $\mu$ Osm/min) & 6 & $11.9 \pm 0.8$ & $11.2 \pm 1.1$ & $13.6 \pm 1.0$ & 6 & $11.2 \pm 0.9$ & $13.2 \pm 2.3$ & $12.4 \pm 1.2$ & 0.97 & 0.41 & 0.28 \\
\hline
\end{tabular}

This article is protected by copyright. All rights reserved 


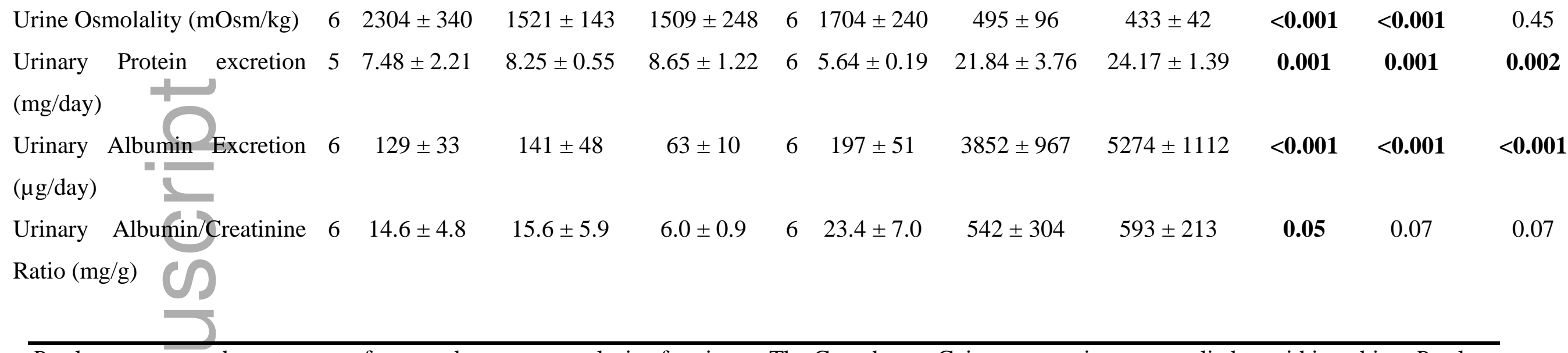

$P$ values represent the outcomes of repeated measures analysis of variance. The Greenhouse-Geisser correction was applied to within-subject $P$ values to correct for eompound asymmetry. ${ }^{49}$ Treat $=$ Treatment

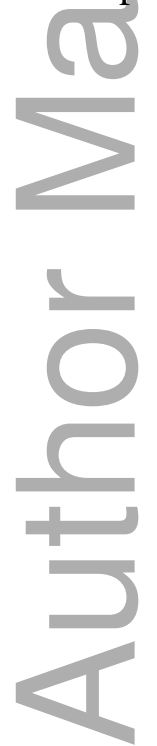

This article is protected by copyright. All rights reserved 
Table 2: Characteristics of rats on day 15 of adenine or vehicle treatment

\begin{tabular}{lccccc} 
Variable & \multicolumn{2}{c}{ Vehicle-treated rats } & \multicolumn{2}{c}{ Adenine-treated rats } & P \\
& n & Day 15 & n & Day 15 & \\
& & & & & \\
& & & & & \\
& 6 & $276 \pm 14$ & 6 & $233 \pm 12$ & 0.04 \\
Body Weight (g) & 5 & $112 \pm 2$ & 3 & $120 \pm 1$ & 0.01 \\
Mean Arterial Pressure (mmHg) & 5 & $368 \pm 25$ & 3 & $317 \pm 11$ & 0.14 \\
Heart Rate (beats/min) & 5 & $1.32 \pm 0.11$ & 5 & $2.38 \pm 0.12$ & $<0.001$ \\
Left Kidney Weight (g) & 5 & $1.23 \pm 0.06$ & 5 & $2.37 \pm 0.16$ & 0.001 \\
Right Kidney Weight $(\mathrm{g})$ & & & & & \\
\hline
\end{tabular}

$P$ values are the outcomes of Student's unpaired t-test.

\section{Figure Legends}

Fig. 1 Micrographs of kidney sections from rats treated with vehicle (a-d) or adenine (e-h), stained with haematoxylin and eosin. Cortical (b, f), outer medullary (c, g) and inner medullary $(\mathrm{d}, \mathrm{h})$ regions are shown. Boxes in (a) and (e) show regions presented in (b-d) and (f-h). Note hypertrophy of the kidney of adenine-treated rats, interstitial expansion and irregular lumen of tubules. Images are typical of two sections taken from each kidney.

Fig. 2 Micrograph of a kidney section from a rat treated with adenine, stained with haematoxylin and eosin. Note the presence of tubular casts (arrows) formed from precipitation of 2,8-dihydroxyadenine.

Fig. 3 Micrographs of kidney sections from rats treated with vehicle (a-d) or adenine (e-h), stained with picrosirius red. Cortical $(b, f)$, outer medullary $(c, g)$ and inner medullary $(d, h)$ regions are shown. Boxes in (a) and (e) show regions presented in (b-d) and (f-h). Note the intense red staining in the kidneys of adenine-treated rats, showing collagen accumulation in the expanded interstitial space. In contrast, vehicle treated rats show faint staining around the glomerulus and considerable staining around arteries and some veins. Images are typical of two sections taken from each kidney. 
Fig.4 Micrograph of kidney sections from rats treated with vehicle (a-d) or adenine (e-h) stained with Masson's trichrome. Cortical (b, f), outer medullary (c, g) and inner medullary $(\mathrm{d}, \mathrm{h})$ regions are shown. Boxes in (a) and (e) show regions presented in (b-d) and (f-h). Note blue staining, representing fibrosis, is ubiquitous in the interstitium of adenine treated rats, but not vehicle-treated rats. Images are typical of two sections taken from each kidney.

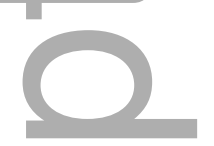

Fig.5 Quantification of fibrosis in the cortex and medulla. Two sections were analysed from each kidney. Values are between-rat mean \pm SEM for adenine- $(n=6$; filled bars $)$ and vehicle- $(\mathrm{n}=4$; opened bars) treated rats. $* * \mathrm{P}<0.01$ for adenine $\mathrm{v}$ control (Student's unpaired t-test).

Fig. 6 Micrographs of kidney sections from rats treated with vehicle (a-d) or adenine (e-h), showing immunohistochemical localisation of pimonidazole adducts. Cortical (b, f), outer medullary (c, g) and inner medullary $(\mathrm{d}, \mathrm{h})$ regions are shown. Note the intense staining in the cortex and medulla of adenine-treated rats. In contrast, vehicle treated rats show minimal staining in the cortex but some staining in the medulla. Images are typical of two sections taken from each kidney.

Fig.7 Relationship between hypoxia and the patency of tubules in kidneys of adenine-treated rats. Twenty non-dilated tubules with a visible lumen (open tubules) and twenty tubules with no visible lumen (closed tubules) were selected from each of the kidney sections stained with Masson's trichrome. These tubules were then identified in serial sections processed for pimonidazole adduct histochemistry. Each tubule was then classified as hypoxic (by the presence of piminidazole stain) or not hypoxic (by the absence of pimonidazole stain). Columns and error bars are between-rat mean \pm SEM of $n=6 . P=0.39$ for comparison between open and closed tubules (Student's paired t-test).

Fig.8 Co-localisation of fibrosis and hypoxia. The micrographs show serial sections from the kidney of a typical adenine-treated rat, stained with Masson's trichrome (a-d), and for pimonidazole adducts (e-h). Cortical (a, e, b, f, c, g) and medullary (d, h) regions are shown. 
Figure 1

\section{Vehicle}

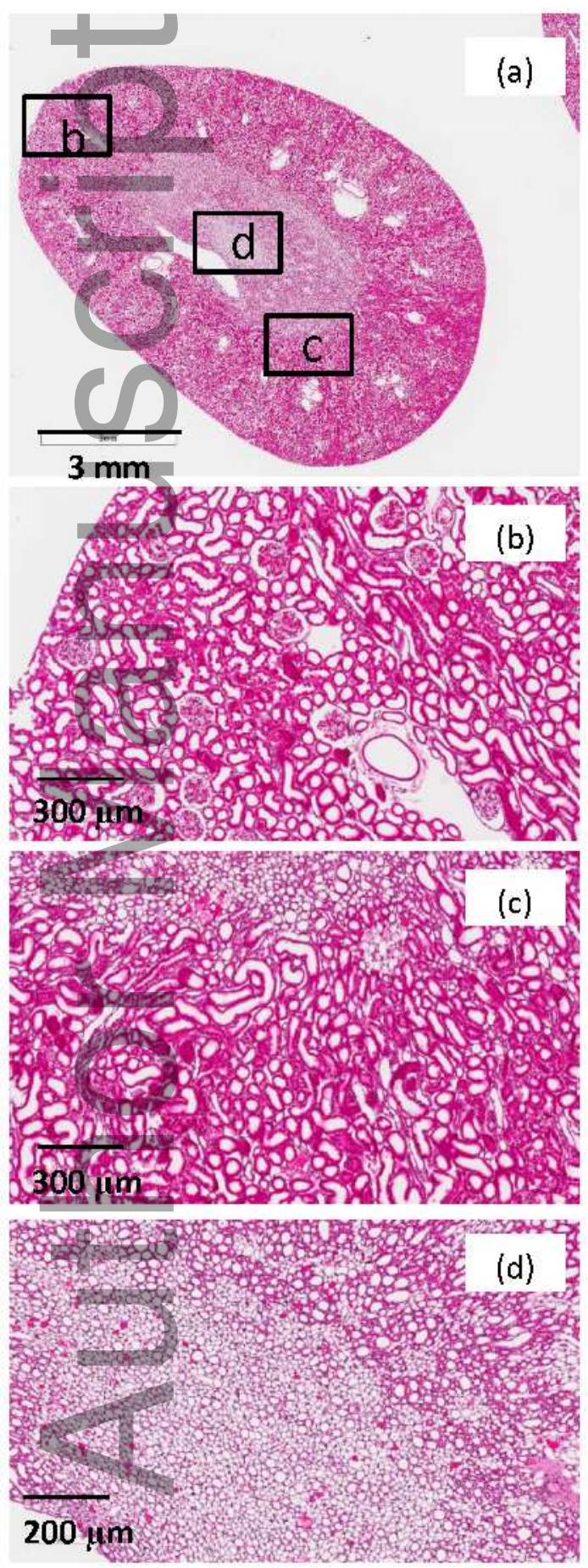

Adenine
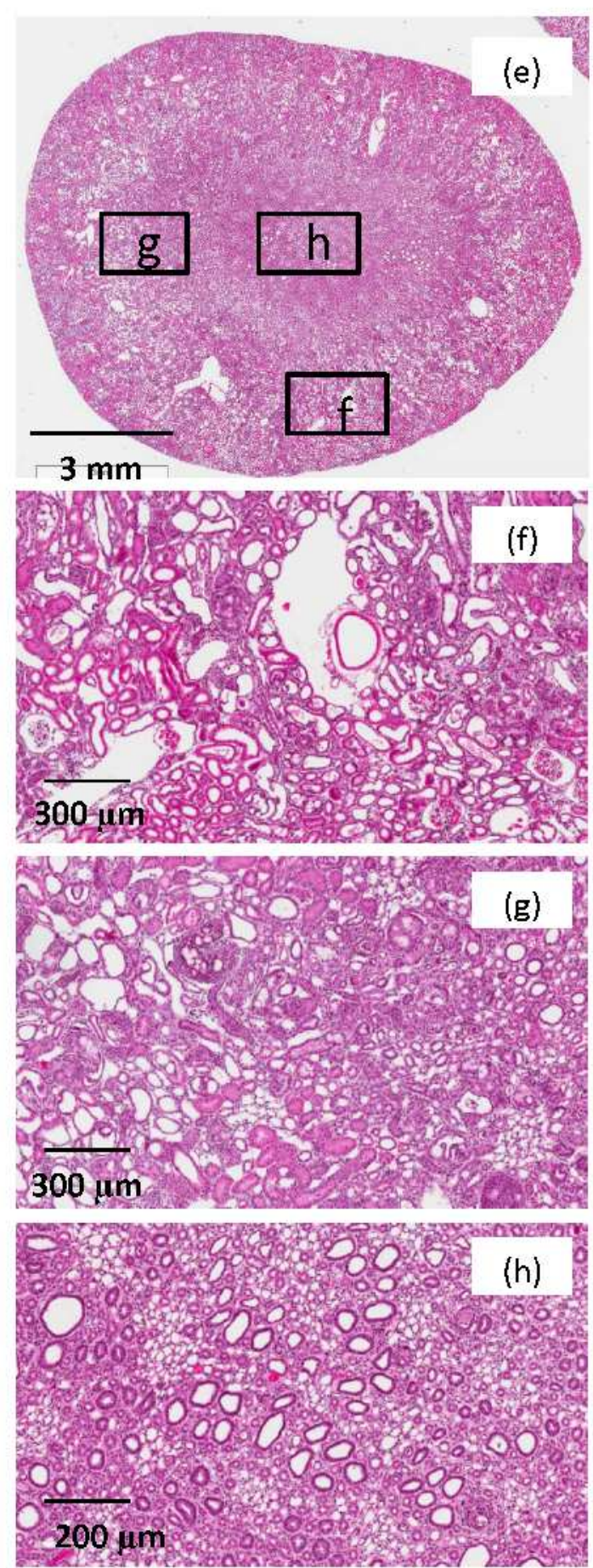

This article is protected by copyright. All rights reserved 
Figure 2
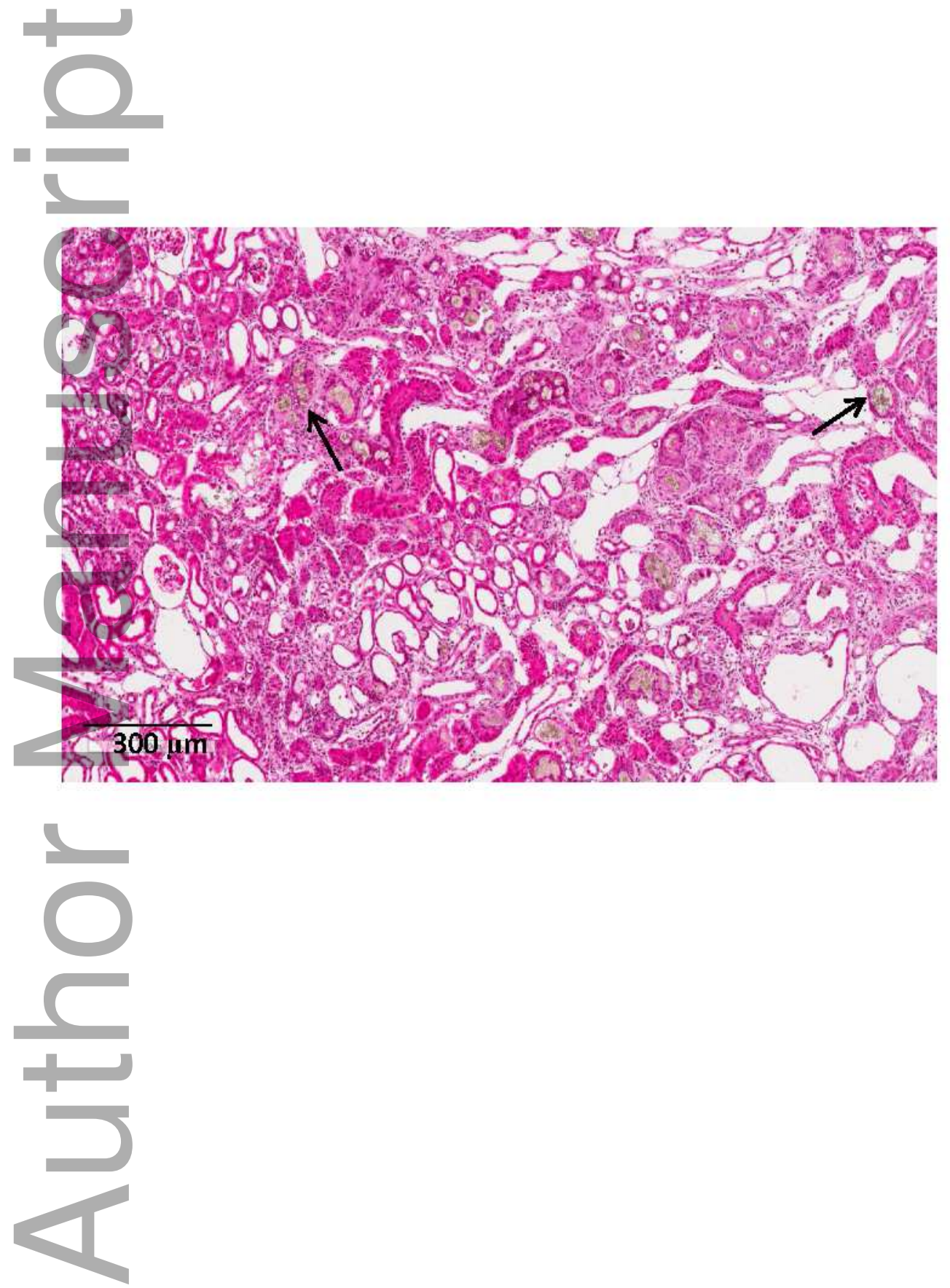

This article is protected by copyright. All rights reserved 


\section{Vehicle}

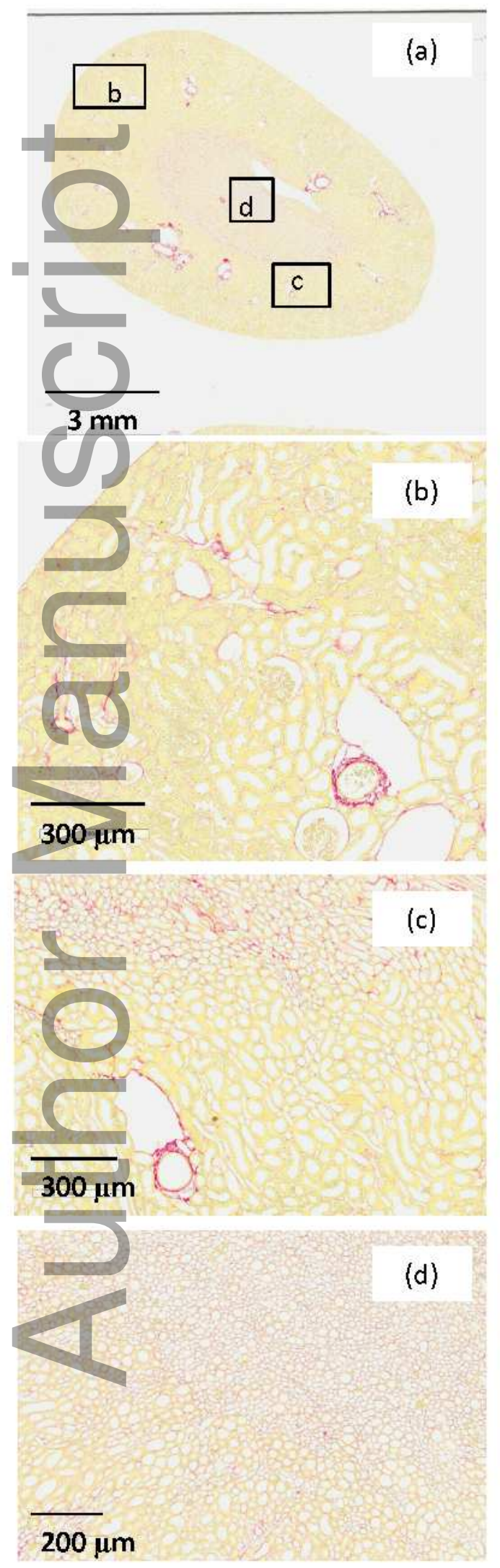

Adenine

Figure 3

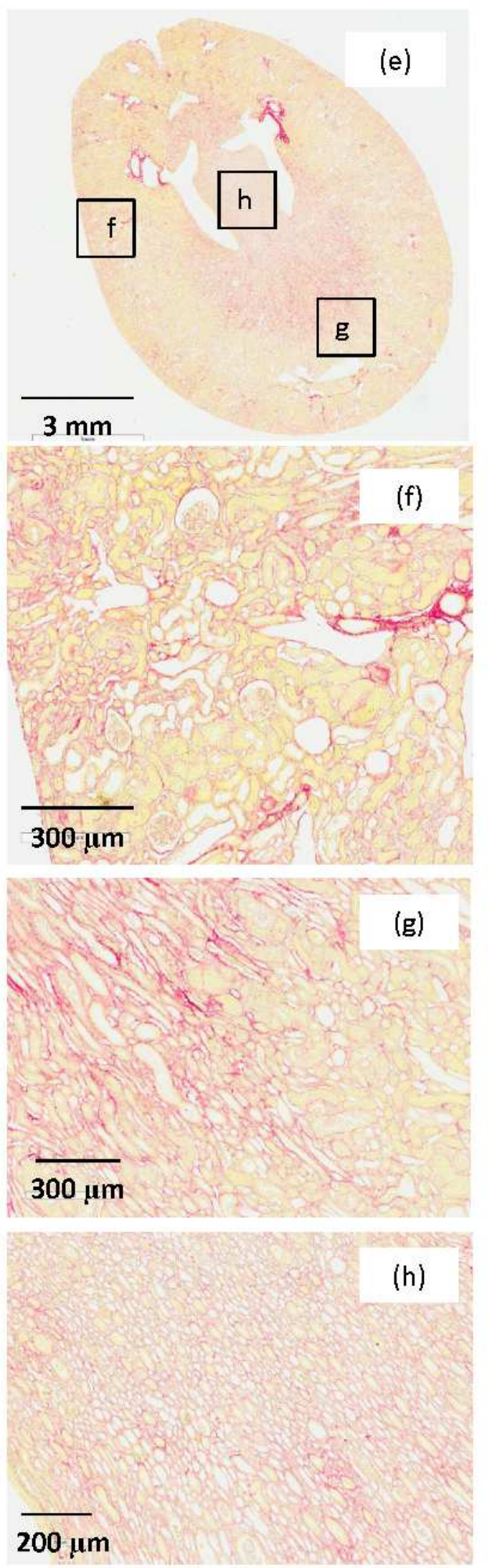

This article is protected by copyright. All rights reserved 
Figure 4

\section{Vehicle}
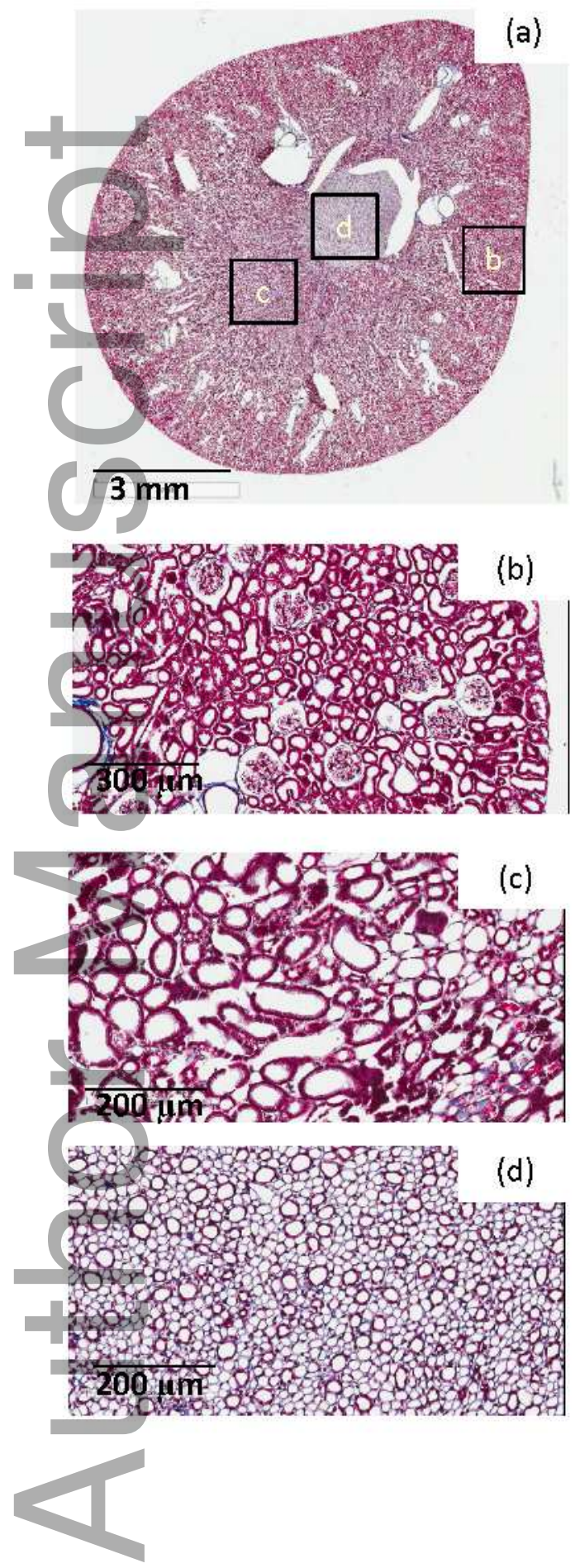

Adenine
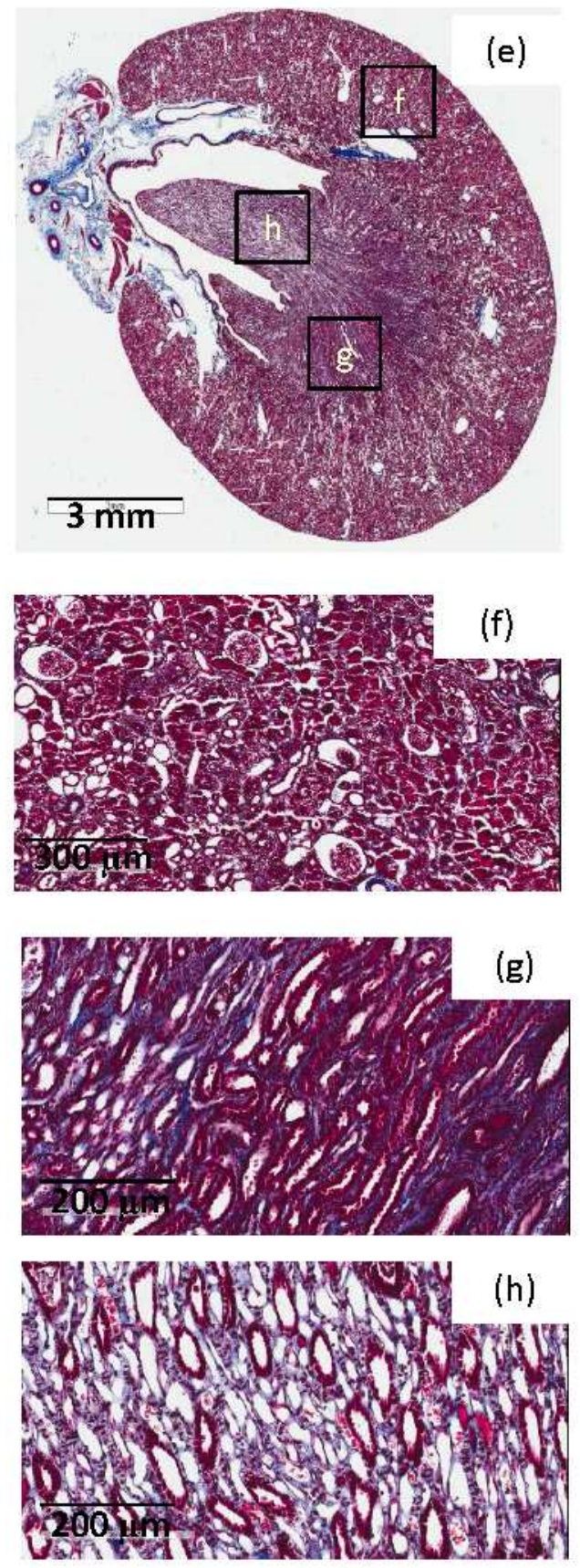

This article is protected by copyright. All rights reserved 
Figure 5

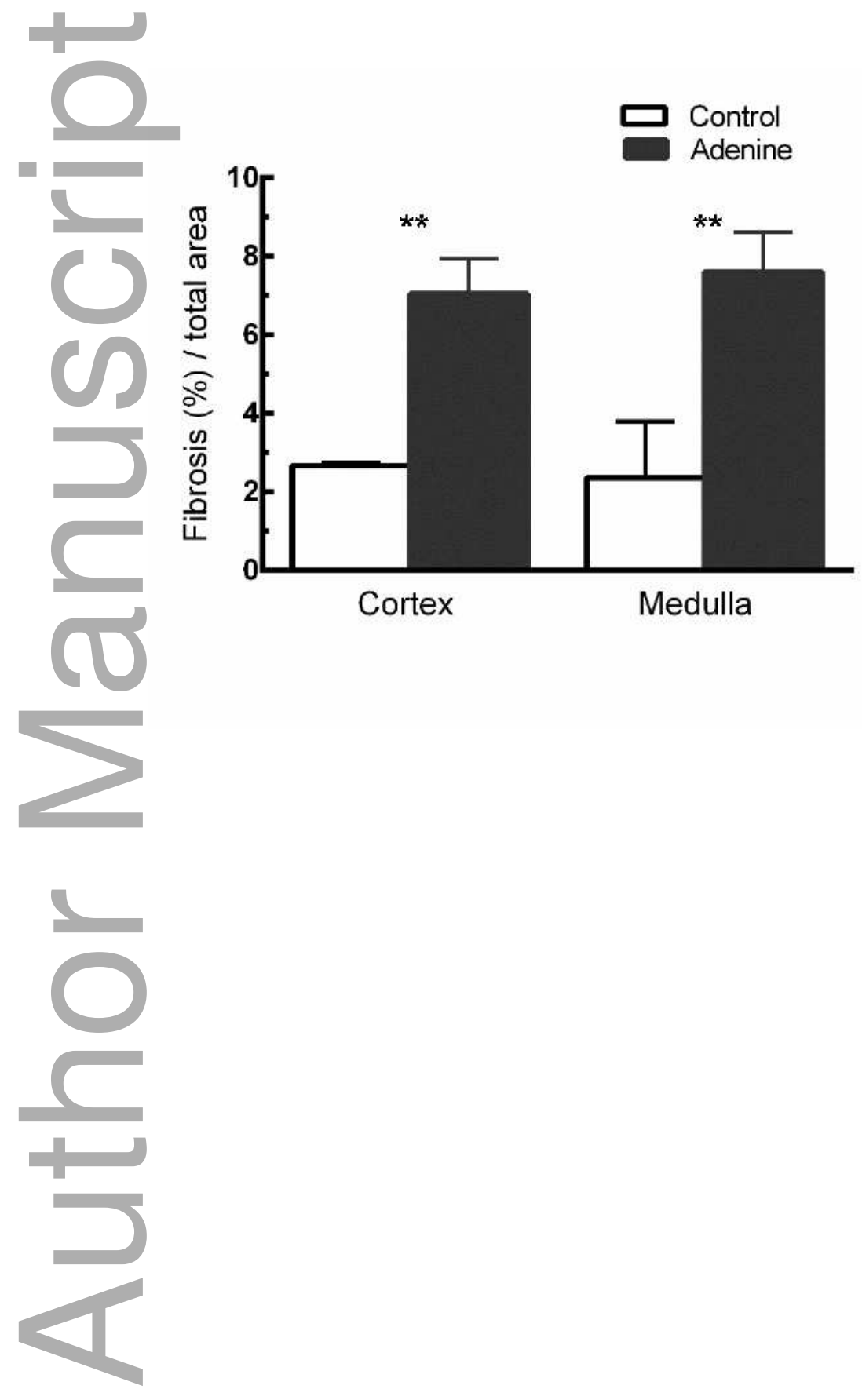

This article is protected by copyright. All rights reserved 
Figure 6

\section{Vehicle}
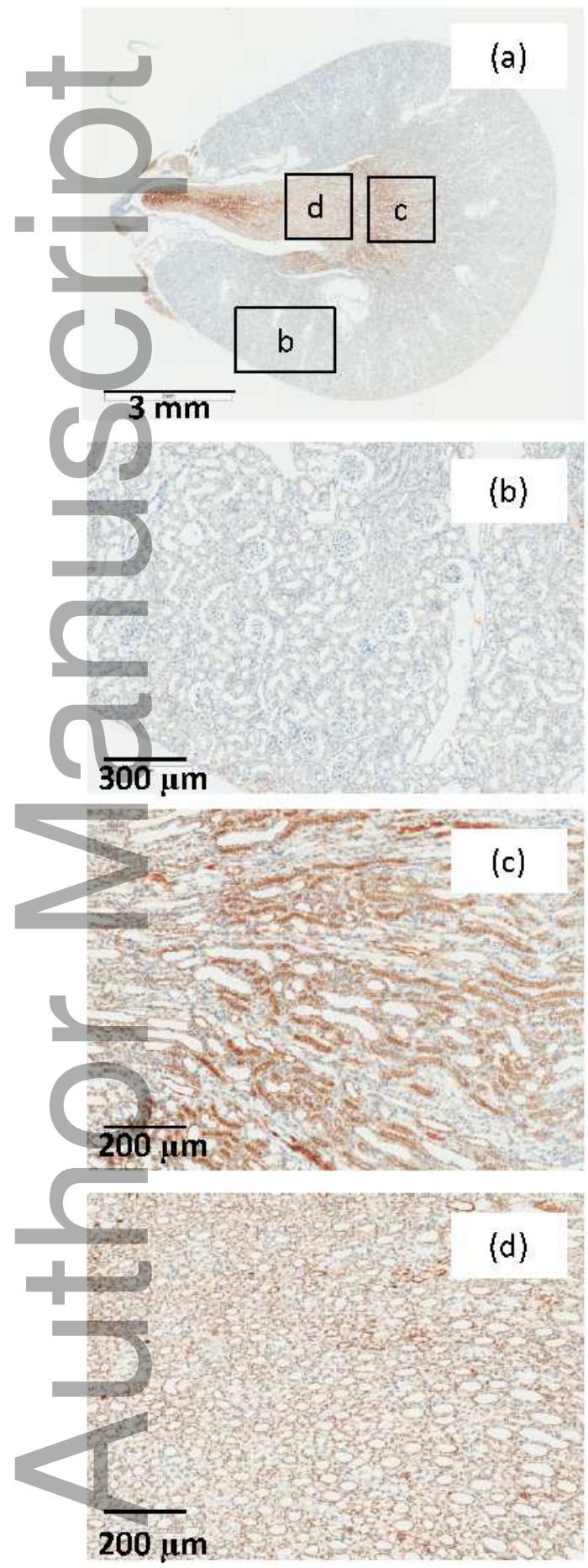

Adenine
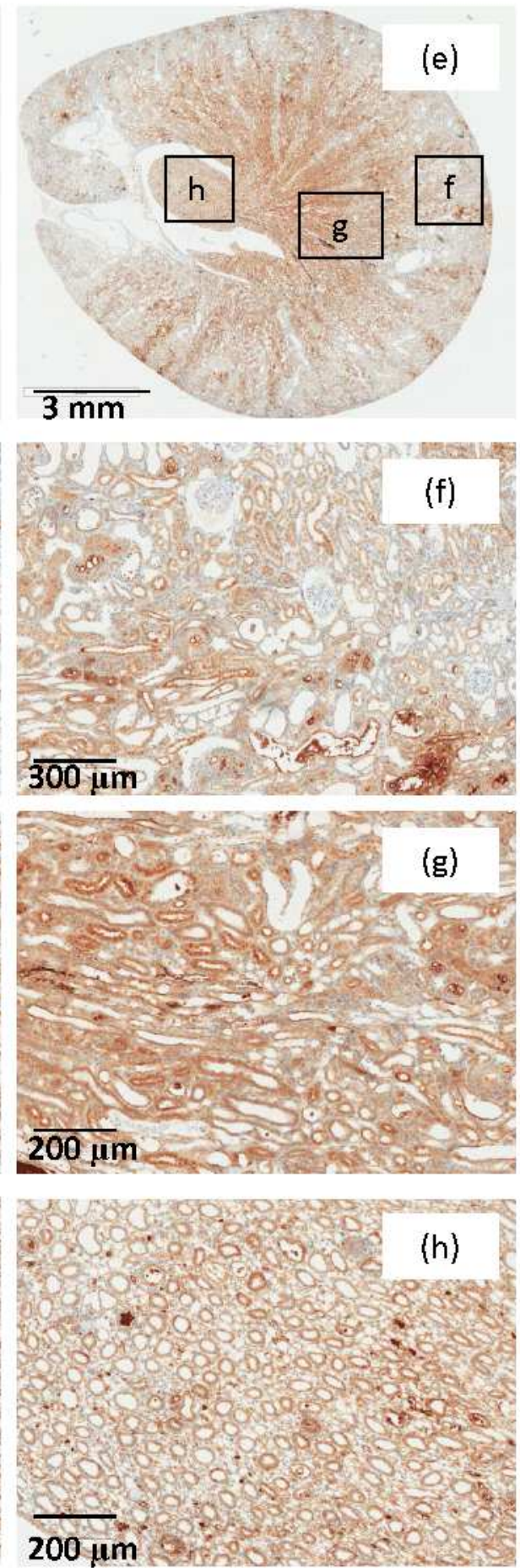

This article is protected by copyright. All rights reserved 
Figure 7

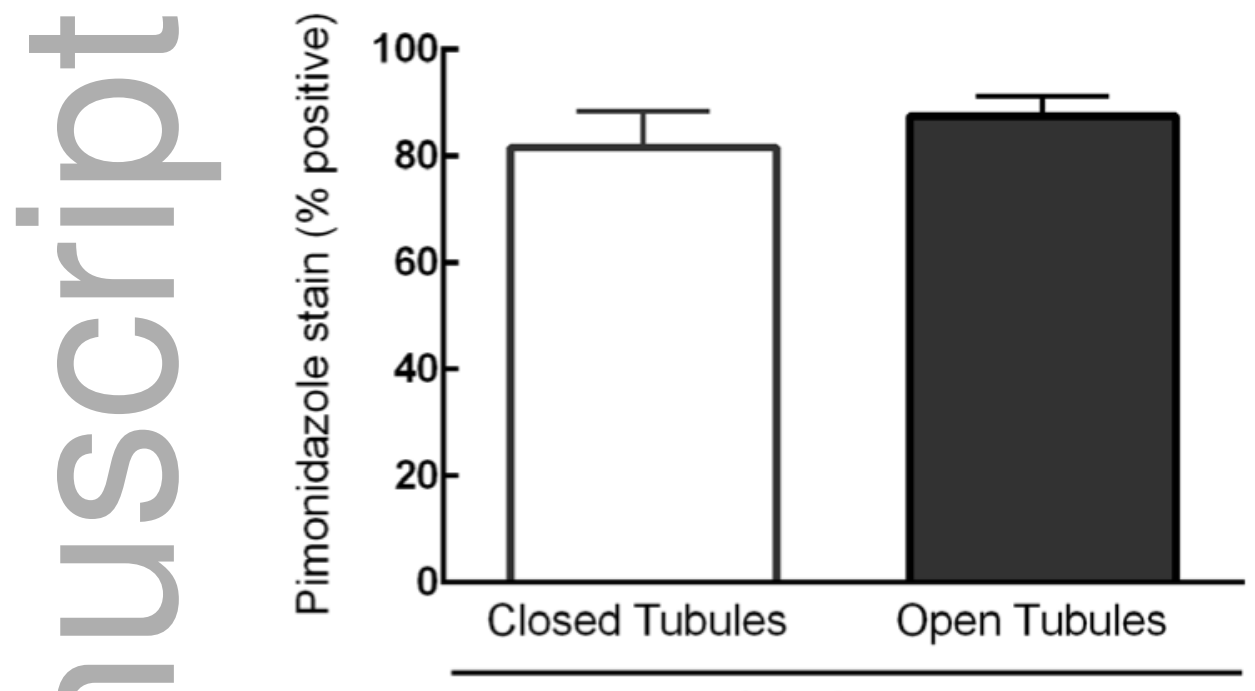

Adenine

This article is protected by copyright. All rights reserved 
Figure 8

Masson's
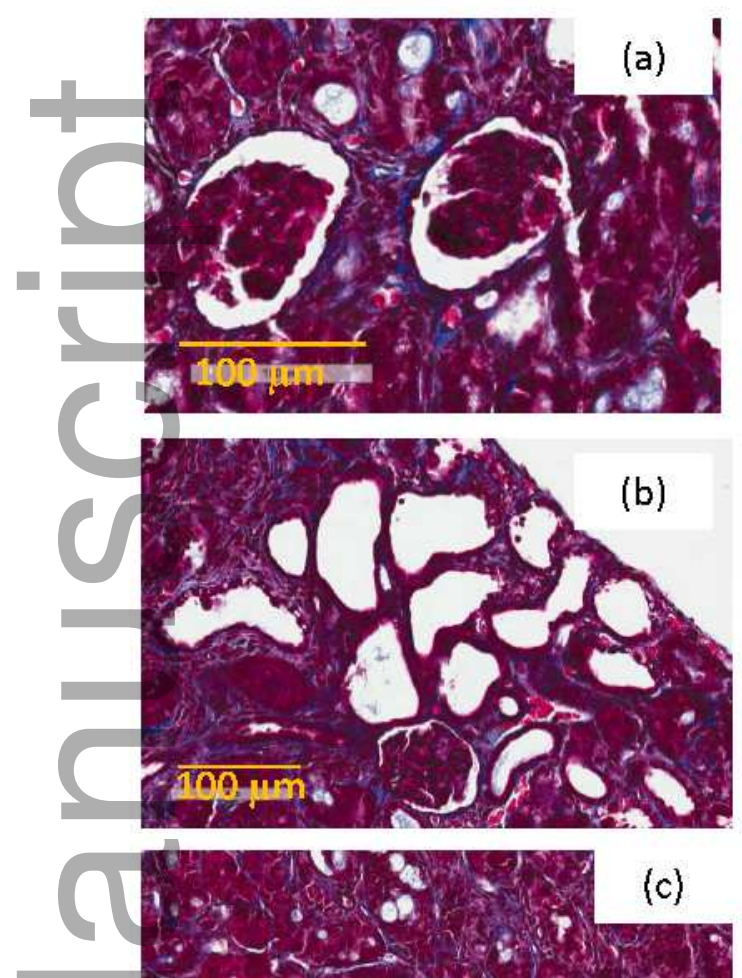

50
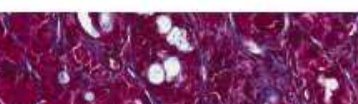

(c)

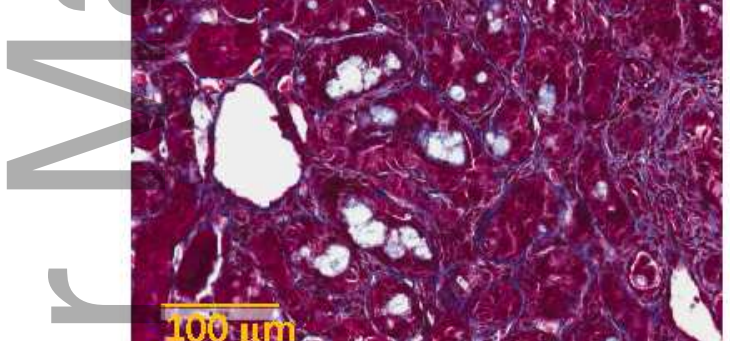

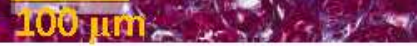

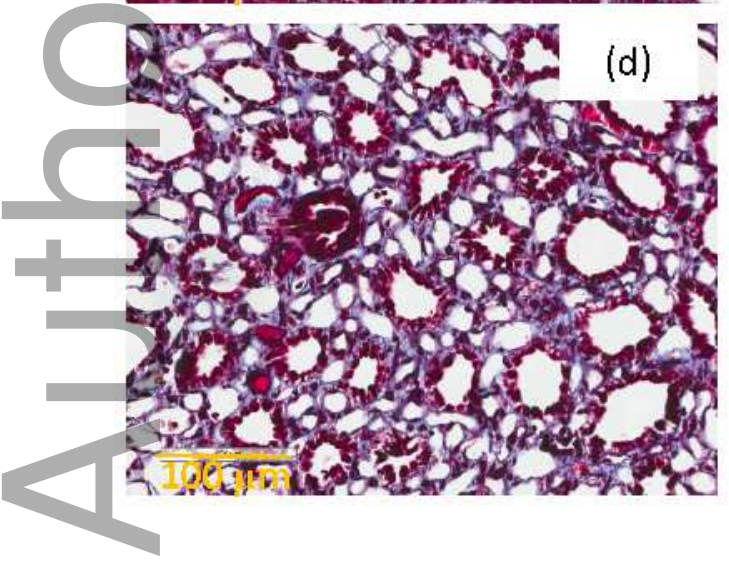

Pimonidazole
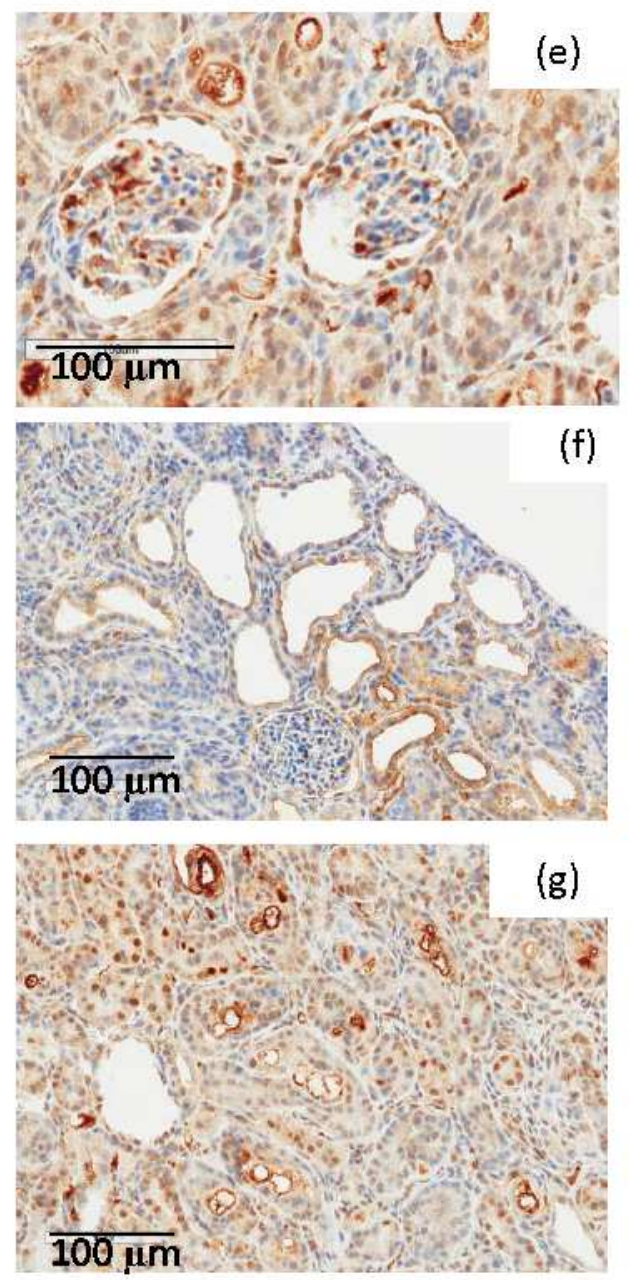

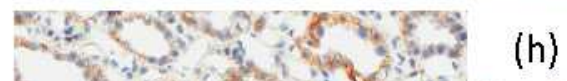
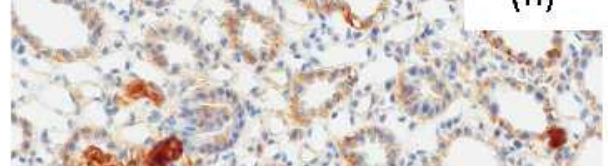

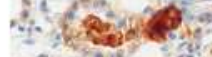

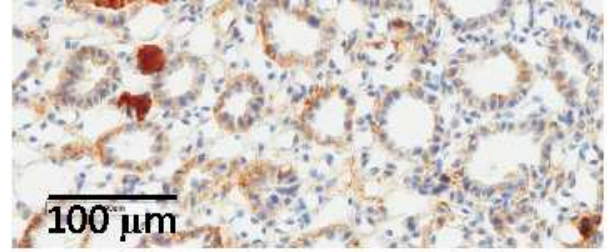

This article is protected by copyright. All rights reserved 


\section{University Library}

\section{- M M N E R VA A gateway to Melbourne's research publications}

Minerva Access is the Institutional Repository of The University of Melbourne

\section{Author/s:}

Fong, D;Ullah, MM;Lal, JG;Abdelkader, A;Ow, CPC;Hilliard, LM;Ricardo, SD;Kelly, DJ;Evans, $R G$

Title:

Renal cellular hypoxia in adenine-induced chronic kidney disease

Date:

2016-10-01

\section{Citation:}

Fong, D., Ullah, M. M., Lal, J. G., Abdelkader, A., Ow, C. P. C., Hilliard, L. M., Ricardo, S. D., Kelly, D. J. \& Evans, R. G. (2016). Renal cellular hypoxia in adenine-induced chronic kidney disease. CLINICAL AND EXPERIMENTAL PHARMACOLOGY AND PHYSIOLOGY, 43 (10), pp.896-905. https://doi.org/10.1111/1440-1681.12621.

Persistent Link:

http://hdl.handle.net/11343/291674 